\title{
CALIDAD DE VIDA, DESEMPEÑO ACADÉMICO Y VARIABLES SOCIODEMOGRÁFICAS EN ESTUDIANTES UNIVERSITARIOS DE SANTA MARTA-COLOMBIA
}

\author{
QUALITY OF LIFE, ACADEMIC PERFORMANCE AND \\ SOCIO-DEMOGRAPHIC VARIABLES COLLEGE STUDENTS IN \\ SANTA MARTA-COLOMBIA
}

\author{
TÍTULO CORTO: CALIDAD DE VIDA, DESEMPEÑO ACADÉMICO Y VARIABLES \\ SOCIODEMOGRÁFICAS EN ESTUDIANTES
}

Ivone Tatiana Brito-Jiménez ${ }^{1}$, Jorge Palacio-Sañudo ${ }^{2}$

Recibido en noviembre 27 de 2015

Aceptado en enero 19 de 2016

\section{RESUMEN}

La investigación permite identificar los efectos de variables sociodemográficas y la calidad de vida en el desempeño académico de 465 estudiantes de la Facultad Ciencias de la Salud de la Universidad del Magdalena. La metodologia utilizada para la obtención de los datos consistió en la aplicación de dos instrumentos a estudiantes de la Facultad Ciencias de la Salud de la Universidad del Magdalena. Un instrumento, de Martha Artunduaga, sobre Variables que influyen en el rendimiento académico de la Universidad y el otro instrumento fue el SF 36 sobre la calidad de vida en salud. Se concluye que no existe relacion entre calidad de vida, factores sociodemográficos y desempeño académico en los sujetos de esta muestra. A la vez, se observó una relacion significativa e inversamente proporcional entre la edad y el desempeño académico al igual que el estrato social y el desempeño académico, presentando que entre más bajo el estrato social mejor desempeño academico tiene y entre menor edad mejor desempeño académico; se relaciona este fenómeno por el hecho de que los estudiantes se enfrentan con fuerza y persistencia a las dificultades percibiendo la educación como una oportunidad de superación de pobreza como eje de la teoría de la movilidad social.

Palabras clave: Características culturales; Calidad de vida; rendimiento; Universitarios; Movilidad social

\section{ABSTRACT}

The research identifies the effects of sociodemographic variables and quality of life in the academic performance of 465 students of the Faculty of Health Sciences of the University of Magdalena. The methodology used for data collection consisted of the application of two instruments to students of the Faculty of Health Sciences of the University of Magdalena, the instrument of Martha Artunduaga on variables that influence the achievement of the

1. Enfermera, magister en Desarrollo Social, Universidad del Norte, Colombia. Correo: ivonebritoj@hotmail.com

2. Doctor en Psicología, Universidad de París X - Nanterre. DEA en Psicología y Maîtrise en Psicología, Universidad de París X. Psicólogo, Universidad del Norte. Coordinador del Doctorado en Psicología de la Universidad del Norte. Investigador del GIDHUM - Grupo de Investigaciones en Desarrollo Humano de la Universidad del Norte, Colombia. Correo: jpalacio@uninorte.edu.co 
University and the instrument SF 36 was the quality of life in health. We conclude that there is no relationship between quality of life, sociodemographic factors and academic performance in the subjects of this sample. While a significant and inverse relationship between age and academic performance as well as the social status and academic performance was observed, showing that the lower the social stratum have better academic performance among younger and better academic performance, are relates this phenomenon by the fact that students are faced with strength and persistence of the difficulties perceiving education as an opportunity to overcome poverty as the core of the theory of social mobility.

Keywords: Traditional Culture; Quality of life; performance; university; Social mobility

\section{INTRODUCCIÓN}

$\mathrm{L}$ a presente investigación pretende correlacionar las variables de calidad de vida, desempeño académico y variables sociodemográficas en estudiantes de ciencias de la salud de la universidad del Magdalena, definidos estos factores como dimensiones en la construcción del conocimiento, pilares fundamentales en el estudio del rendimiento academico en los estudiantes que son un indicador de calidad para las instituciones de educación superior.

A medida que pasan los años algunos estudiantes abandonan el estudio en poco tiempo de haber iniciado el semestre; puede darse por múltiples condiciones tales como: La inadecuada orientación profesional, la falta de un método de estudio, la falta de motivación, dificultades económicas. Los factores o determinantes que influyen en el rendimiento académico de los estudiantes universitarios, son complejos en identificar, dado que se encuentran fuertemente entrelazados; dificultando su delimitación que atribuya efectos claramente inapreciables para ellos ${ }^{1}$. De acuerdo a los hallazgos encontrados algunos autores muestran la gran influencia de determinantes sociodemográficos en estudiantes, tales como: el área geográfica, el nivel socioeconómico y la raza ${ }^{2-5}$.

De acuerdo a este fenómeno, la institución de educación superior debe cuestionarse sobre su eficacia para retener y promover estudiantes, al igual que institución prestadora de un servicio educativo de calidad. De acuerdo al aporte de Garbanzo 6 "El rendimiento académico del estudiantado universitario constituye un factor imprescindible en el abordaje del tema de la calidad de la educación superior, debido a que es un indicador que permite una aproximación a la realidad educativa”.

El rendimiento académico es un indicador de eficacia y calidad educativa, así como un tema que es de interés a nivel académico y social por las variaciones que se han encontrado e impacto al pasar de los años. Es importante destacar que Spinola? "El rendimiento académico ha sido definido como el cumplimiento de las metas, logros u objetivos establecidos en el programa 0 asignatura que está cursando un alumno".

En los estudiantes universitarios el bajo rendimiento académico es producto de varios factores tales como: personales, familiares, académicos y socioculturales; estos suelen permanecer por largos periodos sin que se manifieste, por lo que terminan influyendo en la integridad biopsicosocial del estudiante; reflejándose en el déficit de la atención y memoria, déficit cognitivo; que conlleva a una insuficiente productividad ${ }^{8}$.

No obstante, de acuerdo al análisis de la conceptualización se puede decir que es la unión de factores endógenos y exógenos del estudiante, el cual genera éxito o fracaso académico, valor agregado a la calidad y gestión de las instituciones de educación superior. Es importante resaltar los factores que caracterizan el rendimiento académico como influyente en la calidad de vida de los estudiantes universitarios dado por el desarrollo en cada semestre cursado. El logro académico de los estudiantes universitarios está dado por los múltiples elementos que construyen el rendimiento como valor agregado al desempeño del individuo $0^{9,10}$. 
A la vez se pueden relacionar o asociar factores internos y externos en el rendimiento academico asi como lo menciona Araya y Sierra ${ }^{11}$ "Es clara la asociación directa entre los factores externos o extrinsecos como la pobreza, fracaso educativo y delincuencia”.

Teniendo en cuenta los factores externos o extrinsecos se tiene en cuenta la institución educativa superior asi como lo menciona Latiesa ${ }^{12}$ "La influencia de los factores de la institución educativa superior juegan un papel importante dado que sus características eficaces dependen de cada institución depende de sus particularidades”.

Como factor intrínseco o interno tienen las personales o propias asi como es citado por los modelos de las investigaciones de Salonava et $\mathrm{a}^{13}$ "teniendo en cuenta la teoría social Cognitiva, se refiere a las capacidades del individuo para estructurar y establecer sus prioridades que le faciliten obtener su máximo logro, evidenciándola como la habilidad del ser humano para alcanzar sus metas".

Teniendo en cuenta la variable de calidad de vida y sus sub escalas se plantean algunas condiciones a partir de la revisión bibliográfica se tiene el aporte de $\mathrm{BID}^{14}$. El Banco Interamericano de Desarrollo apunta a que "todo el mundo desea lograr una mejor calidad de vida, aunque pocas personas pueden definir con precisión el objetivo de su búsqueda”.

$\mathrm{Y}$ desde la perspectiva de Quintero et $\mathrm{al}^{11}$ en la conceptualización y utilización del término de calidad de vida relacionada con la salud desde (CVRS) desde 1988 "Este término se usa para indicar las medidas centradas en la salud y en el campo de resultados de salud donde se tiene en cuenta la opinión de las personas respecto a su salud".

Desde la perspectiva de O’Boyle ${ }^{15}$ "plantea que la objetividad en la evaluación está dada por el análisis de los factores internos y externos como: la salud, el ambiente, los ingresos, vivienda; entre otros indicadores que facilitan la medición de la Calidad de Vida; además se vinculan juicios objetivos conllevando a que la Calidad de Vida sea percibida desde otra perspectiva se observa complejamente difícil de pronosticar".

Un punto importante en el aspecto de calidad de vida está orientado a la capacidad y al proceso de adaptación del sujeto ante el mantenimiento de la salud, y el mantenimiento del nivel de satisfacción constante, a pesar de los cambios ambientales ${ }^{16}$.
Se realiza un análisis de las sub escalas de Calidad de Vida y la relación con factores del rendimiento académico y los factores sociodemográficos como parámetro de evaluación en los estudiantes del área de la salud a nivel universitario. Por esta razón se realizará correlación de estos elementos vistos como tema de interés a nivel social y educativo.

Este tema es visto como prioridad aplicable porque de acuerdo a la condición en la cual se desarrolla el estudiante visto desde las variables depende su desempeño académico en la construcción de su profesión y el futuro del desempeño del profesional de la salud representada por los estudiantes de la Universidad del Magdalena Facultad de ciencias de la salud.

La investigación se fundamenta en las Sub escalas de Calidad de vida en salud dirigidos a estudiantes universitarios como actor principal en el proceso de enseñanza aprendizaje por tal razón se analiza y se observa cómo se transforma este concepto de acuerdo al contexto en el cual de desarrolla el individuo y se origina de las experiencias vividas de cada ser humano teniendo en cuenta los diversos factores o determinantes que influyen; por esta primicia se hace útil y necesario analizar los factores sociodemográficos que pueden o no llegar a generar un impacto y posteriormente conllevar a la deserción estudiantil que representa indicador negativo de gestión y calidad de la Institución Educativa.

Para ello se aplicaron instrumentos en donde se analiza factores asociados, factores que inciden en el rendimiento académico utilizado por Marta Artunduaga en su tesis doctoral denominado Variables que influyen en el rendimiento academico de la Universidad y el instrumento que mide la calidad de vida denominado SF 36 a los estudiantes de los programas de odontología, enfermería y medicina de la facultad ciencias de la salud de la Universidad del Magdalena. Por último, hallar la correlación que existe entre estas variables.

\section{MATERIALES Y MÉTODOS}

\section{Diseño}

El diseño de esta investigación fue Correlacional ya que tiene la finalidad de "medir la fuerza de asociación o relación que existe entre dos o más conceptos o variables en un momento determinado" de acuerdo a lo referencia por Hernández, et $\mathrm{al}^{17}$. Las variables a tener en cuenta son los factores sociodemográficos, desempeño Académico y Calidad de Vida. 


\section{Población y muestra}

La población de estudiantes de la Facultad Ciencias de la Salud es de 2000 estudiantes y la muestra inicial fue de 465 sujetos entre mujeres y hombres, discriminadamente fueron 119 hombres y 347 mujeres; los semestres escogidos para el estudio fue de Segundo a Noveno semestre de los programas enfermería, medicina y odontología; dado que son estudiantes que se encuentran permanentemente en el campus universitario y pueden dar una opinión clara sobre los ítems evaluados; a la vez se encontraron variedad en los rangos de edad a partir de los 15 hasta 35 años; importante mencionar que los estudiantes de primer semestre fueron excluidos del estudio porque de acuerdo al modelo educativo de la Universidad pertenecen al Ciclo General y las asignaturas son iguales para todos los estudiantes de las diferentes Carreras que a la vez no son propias del área de la Salud y con relacion a los semestre se excluyó a los estudiantes de Décimo porque están en otras ciudades realizando de las Practicas Profesionales.

La cantidad de instrumentos aplicados por semestres en los diferentes programas académicos fue asi: segundo semestre 55 , tercer semestre 60 , tercer semestre 46 , cuarto semestre 50 , quinto semestre 66 , sexto semestre 66, séptimo semestre 66, octavo semestre 61 y noveno 61.

\section{Técnicas}

Se realizó una técnica de muestreo probabilístico estratificado proporcional (MEP) siendo los estratos definidos por las carreras que se imparten en la Facultad de Ciencias de la Salud.

Inicialmente se visitaron a los diferentes grupos de estudiantes de segundo a noveno semestre, se realizó explicación de la importancia de la investigación, posteriormente se entregaron las instrucciones de diligenciamiento del instrumento. Luego se entrega el instrumento en donde respondían inmediatamente, bajo la supervisión y orientación de la investigadora; teniendo en cuenta la primicia de "conjunto de preguntas respecto a una o más variables a medir" ${ }^{\prime 1}$.

\section{Instrumentos}

Los Instrumentos utilizados luego de haber realizado revisión exhaustiva de la literatura se concluyó utilizar el instrumento de Martha Artunduaga Murillo aplicado en su tesis doctoral denominado variables que influyen en el rendimiento académico en la universidad realizado en la Universidad Complutense de Madrid por Artunduaga ${ }^{18}$ como tesis de su doctorado. Este instrumento consta de preguntas las cuales están agrupadas por factores tales como: Factores Sociodemográficos, Factores Personales, Factores Académicos, Factores Institucionales, satisfaccion, anticipación al futuro académico y profesional y autoconcepto académico.

El Cuestionario de Salud SF 36 es el segundo instrumento utilizado el cual consta de 36 ítems, que analizan las 8 dimensiones del estado de salud: función física; función social; limitaciones del rol: de problemas físicos; limitaciones del rol: problemas emocionales; salud mental; vitalidad; dolor y percepción de la salud general, arrojando como resultado un bajo o alta calidad de vida en salud. El Alfa de Crombach es positiva y alta estando en general en $0.86^{19,20}$.

\section{Métodos de análisis de datos}

Para el procesamiento de datos y análisis estadísticos se utilizó del software SPSS (Statistical Package for the Social Sciences) 18 la cual facilita la organización de los datos en una forma estructurada y posteriormente permite el análisis con diversas técnicas estadísticas

\section{Declaración de aspectos éticos}

De acuerdo a lo dispuesto en la Resolución N 008430 del 4 de octubre 1993 del Ministerio de Salud, el trabajo de grado es clasificado de acuerdo a los estándares de riesgo como Investigación con riesgo mínimo, en donde se utilizó una metodología de investigación en donde se observa, se aplicaron instrumentos, se registró e identificaron variables sociodemográficas, aspectos de salud y desempeño académico en estudiantes universitarios, para analizar la correlación que existe entre estas variables. Dada las circunstancias que el proyecto es realizado con la intervención de personas, se han tenido en cuenta los riesgos, y se garantiza la protección de los sujetos; basados en el capítulo 1 sobre los aspectos éticos de la investigación en seres humanos.

La investigación se rige en el respeto a la dignidad de las personas, su libertad y autonomía y con mayor importancia se preservó la privacidad de los participantes. Teniendo en cuenta este aspecto antes de aplicar los instrumentos se entregó consentimiento informado; partiendo de esta primicia en donde la información suministrada por los sujetos evaluados se basa en su percepción y autorización. 
Es relevante destacar que esta investigación respetará la propiedad intelectual, de los que ha investigado previamente en el tema, de esta manera los investigadores se acogen al reglamento de propiedad intelectual en la construcción y publicación del mismo.

A la vez se tiene en cuenta las normas éticas de la Declaración de Helsinki de 1975 que representa la asociación médica mundial en la cual se definen los principios éticos para investigación médica en seres humanos, en donde la base de la investigación sea promover y velar por las condiciones de los sujetos.

\section{RESULTADOS}

\section{Variables sociodemográficas y desempeño académico}

Los estudiantes de Ciencias de la Salud tomados como muestra se encuentran matriculados en los programas de Medicina 160 estudiantes, en el Programa de Enfermería 170 estudiantes, en el Programa de Odontología 135 estudiantes; el 74.6\% (347) son mujeres y el 25.4\% (118) son hombres para un total de 465 estudiantes; de acuerdo a las edades el 39.4\% (183) corresponde a jóvenes entre 20 y 22 años y 36,3\% (169) entre 17 y 19 años; de estos estudiantes se encuentran en estrato tres un $22 \%$ (103), dos $36.8 \%$ (171) y estrato uno $36.1 \%$ (168), solo un $5 \%$ es de estrato cuatro o mayor.

\section{El apoyo familiar}

Representa para los estudiantes universitarios uno de los factores que permite conseguir los objetivos académicos. Los estudiantes en un $84 \%$ están muy de acuerdo en que su familia los apoya para culminar con éxito la carrera, y solo cinco estudiantes no perciben este apoyo; Indican que su familia confía en su futuro como profesionales un $97 \%$ y solamente dos personas consideran que su familia tiene dudas sobre esto; Por otro lado, consideran que su familia está de acuerdo que obtendrá buenos resultado académicos durante su carrera $67.5 \%$ (314), y un 54\% considera que en sus casas cuentan con un espacio para estudiar, pero un $18,7 \%$ indican no tener este espacio.

\section{Satisfacción con el ambiente universitario}

La satisfaccion del estudiante universitario tiene más fuerza y auge sobre todo en este siglo donde se apunta a la calidad y la formación integral en las instituciones educativas a nivel superior; a partir de esta primicia se evita la deserción estudiantil y se alcanza el éxito academico. Con respecto a la percepción de los estudiantes sobre el ambiente universitario manifiestan que los docentes trabaján con profesionalismo 48.0\% (223), accesibilidad de docentes $44.5 \%$ (207), el personal administrativo atiende las necesidades de los estudiantes, 43.7\% (203), la universidad cuenta con espacios recreativos y culturales $42.2 \%(196)$.

\section{Desempeño académico}

Con respecto al rendimiento académico durante el bachillerato, un $82 \%$ los estudiantes consideran que fue muy bueno su desempeño, y un $95.9 \%$ no repitió materias durante el bachillerato y el $4.1 \%$ si repitieron algún curso. Los problemas más importantes para su desempeño en ese momento se relacionan con problemas familiares y académicos, pero en un bajo porcentaje, siendo la gran mayoria estudiantes con un buen desempeño y apoyo familiar.

El rendimiento académico observado desde la trayectoria estudiantil en la universidad, los estudiantes manifiestan que el 69.0\%(321) han recibido honores; han perdido asignaturas en la carrera actual $52.3 \%$ (243) de estos estudiantes el $60.3 \%$ han perdido entre uno y dos asignaturas y el $38.9 \%$ han perdido entre tres y catorce asignaturas. Por otra parte, los estudiantes opinan que los factores externos por los cuales no obtuvieron buenos resultados fue determinado por la metodología del docente en un $25 \%$, en cuanto a la forma de evaluación del profesor las opiniones están dividas; en un $21.6 \%$ de los estudiantes opinan que la metodología del profesor no influye y el $17.9 \%$ opina que si influye en la obtención de buenos resultados académicos.

Los estudiantes no atribuyen la falta de conocimiento de la asignatura $32.9 \%$ para la obtención de buenos resultados y la dificultad en comprender los temas no se atribuye al bajo rendimiento en un en $33.7 \%$, y en cuanto al tiempo dedicado al estudio con un $25.8 \%$ los estudiantes muestran una fuerte tendencia en que la pérdida de asignaturas no se da por la falta de dedicación.

Para realizar una síntesis sobre el desempeño académico de los estudiantes universitarios es importante destacar que el 37,2 (173) \% tuvo en desempeño alto, el 31.8\% (148) corresponde a estudiantes con desempeño medio alto; el $10.5 \%$ ( 49) corresponde a desempeño medio bajo, mientras el 20.4\% (95) de los estudiantes tienen un rendimiento académico bajo (Tabla 1). 
Tabla 1. Nivel de desempeño de estudiantes universitarios.

\begin{tabular}{|c|c|c|c|c|c|}
\hline \multicolumn{2}{|c|}{} & Frecuencia & Porcentaje & Porcentaje válido & Porcentaje acumulado \\
\hline \multirow{4}{*}{ Válido } & Bajo & 95 & 20,4 & 20,4 & 20,4 \\
\cline { 2 - 6 } & Medio bajo & 49 & 10,5 & 10,5 & 31,0 \\
\cline { 2 - 6 } & Medio alto & 148 & 31,8 & 31,8 & 62,8 \\
\cline { 2 - 6 } & Alto & 173 & 37,2 & 37,2 & 100,0 \\
\cline { 2 - 6 } & Total & 465 & 100,0 & 100,0 & \\
\hline
\end{tabular}

\section{Calidad de vida}

Al realizar el análisis de las subescalas de Calidad de Vida, se observa que en general, la salud física es la que presenta una mejor evaluación $(76,9 \%)$ contra la salud mental con $66,6 \%$. Al parecer el componente físico u orgánico sale mejor evaluado por los sujetos, lo cual se corresponde con la sub escala de funcionamiento físico que tiene la valoración más alta y positiva de todas $(93,3 \%)$. Mientras que la sub escala de energía o fatiga es la más baja de todas $(58,1 \%)$ y puede estar afectando la percepción de la salud mental. Además, la sub escala de problemas emocionales, que se relaciona con la salud mental, es la de mayor dispersión en toda la escala, indicando que los estudiantes de esta muestra presentan grandes diferencias a nivel emocional, es decir unos muy bien y otros muy mal (Tabla 2).

Tabla 2. Sub escalas de Calidad de Vida - SF 36.

\begin{tabular}{|c|c|c|c|c|c|c|c|}
\hline & \multirow{2}{*}{ Media } & \multirow{2}{*}{ Mediana } & \multirow{2}{*}{ Moda } & \multirow{2}{*}{$\begin{array}{c}\text { Desviación } \\
\text { estándar }\end{array}$} & \multicolumn{3}{|c|}{ Percentiles } \\
\cline { 7 - 8 } & & & & 25 & 50 & 75 \\
\hline Funcionamiento físico & 93,355 & 100,000 & 100,0 & 11,8145 & 90,000 & 100,000 & 100,000 \\
\hline Rol físico & 81,075 & 100,000 & 100,0 & 33,6280 & 75,000 & 100,000 & 100,000 \\
\hline Problemas emocionales & 69,606 & 100,000 & 100,0 & 39,4340 & 33,333 & 100,000 & 100,000 \\
\hline Energía/Fatiga & 58,118 & 55,000 & 50,0 & 16,5785 & 45,000 & 55,000 & 70,000 \\
\hline Salud mental & 64,120 & 64,000 & 48,0 & 17,9497 & 48,000 & 64,000 & 80,000 \\
\hline Funcionamiento social & 74,892 & 75,000 & 100,0 & 22,6982 & 62,500 & 75,000 & 100,000 \\
\hline Enfermedad corporal & 68,263 & 67,500 & 100,0 & 23,5834 & 55,000 & 67,500 & 90,000 \\
\hline Salud general & 65,301 & 65,000 & 70,0 & 18,5090 & 50,000 & 65,000 & 80,000 \\
\hline Salud Física General & 76,999 & 81,250 & 86,3 & 16,1333 & 68,125 & 81,250 & 88,750 \\
\hline Salud Mental General & 66,684 & 68,125 & 92,0 & 18,5017 & 53,583 & 68,125 & 81,063 \\
\hline
\end{tabular}

En cuanto a la salud física general y mental en estudiantes universitarios, se observa que la salud física se presenta con mejores indicadores que la salud mental. En salud física general existe una media de 77 y una desviación estándar de 16.133 y en cuanto a la salud mental general tiene una 138 media de 66.68 y una desviación estándar de 18.502.

Eso indica que es más dispersa la salud mental general que la salud física general, por lo tanto, hay más personas que tienen una salud mental baja y alta y en cuanto la salud física general la mayoría de personas están muy bien en esta condición (Figura 1 y 2).

Por último, se observa que la opinión de los estudiantes con respecto al nivel de desempeño está relacionada con la edad; entre más jóvenes los estudiantes, el nivel de desempeño es mejor y entre más adultos menor nivel de desempeño presentan (Figura 3 y Tabla 3). 


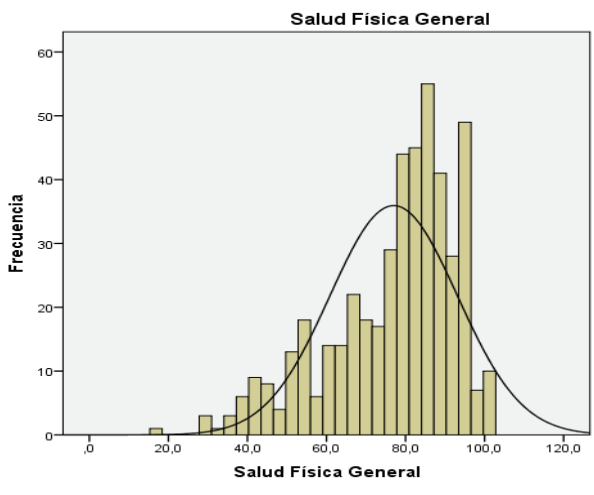

Figura 1. Salud física general.

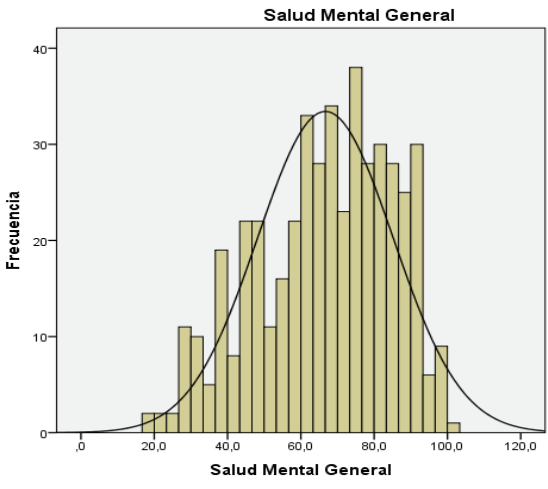

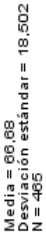

Figura 2: Salud mental general.

Tabla 3. Edad - alto y bajo desempeño académico.

\begin{tabular}{|c|c|c|c|c|c|c|}
\hline \multicolumn{2}{|c|}{} & N & Media & $\begin{array}{c}\text { Desviación } \\
\text { estándar }\end{array}$ & F & Sig. \\
\hline \multirow{3}{*}{ Edad } & Bajo desempeño & 144 & 2,99 &, 824 & 6,126 &, 014 \\
\cline { 2 - 7 } & Alto desempeño & 321 & 2,80 &, 753 & & \\
\cline { 2 - 7 } & Total & 465 & 2,86 &, 779 & & \\
\hline \multirow{2}{*}{$\begin{array}{c}\text { Estrato social al } \\
\text { cual pertenece }\end{array}$} & Bajo desempeño & 144 & 2,03 &, 784 & 4,689 &, 031 \\
\cline { 2 - 7 } & Alto desempeño & 321 & 1,86 &, 789 & & \\
\cline { 2 - 7 } & Total & 465 & 1,91 &, 791 & & \\
\hline
\end{tabular}

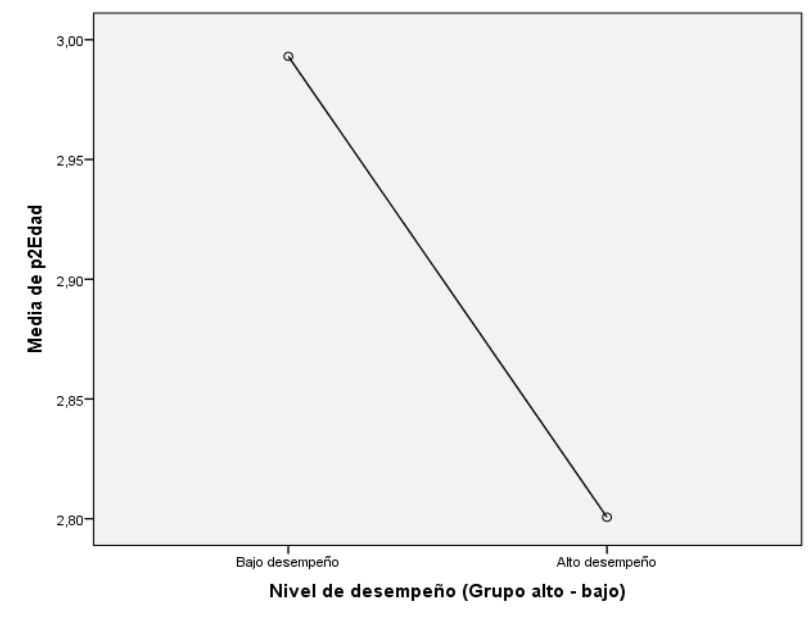

Figura 3. Edad/ nivel de desempeño.

\section{DISCUSIÓN}

En esta investigación no se probaron las hipótesis de trabajo, ya que se esperaba que el desempeño académico se viera afectado negativamente por la calidad de vida y los factores socioeconómicos de los estudiantes. Esta condición está dada por las características propias de la población en estudio, en la cual influyen otros factores intrínsecos que le hacen un contrapeso a los factores negativos, tales como el deseo de superación y la motivación de alcanzar sus metas, al igual que el deseo de salir adelante. En la mayoría de las universidades públicas, los estudiantes en general tienen un excelente promedio y vienen de familias con bajos recursos económicos, por lo cual la educación es una puerta de escape a la pobreza, convirtiéndose de esta manera en un ascensor social que les permite a los jóvenes soñar con un futuro mejor. De allí que los jóvenes desde niños han aprendido a sortear estas situaciones difíciles, desarrollando comportamientos resilientes a las situaciones adversas.

Estos resultados se analizan desde la teoría de la movilidad social y el desarrollo económico. Esta situacion particular es reforzada por la transición demográfica ${ }^{21}$ quienes mencionan que se trazan los límites de la "ventana de oportunidades" que se les presentan a los jóvenes de bajos recursos 0 en condicion de pobreza cuando acceden a estudios superiores como ascensor social. A la vez se refiere al horizonte que perciben los jóvenes para poder mejorar su situación económica y social y subir en la estructura social. 
Los jóvenes sujetos de esta investigación, tienen en general un alto desempeño académico, pero sobre todo aquellos que son más jóvenes son los que mejor desempeño presentan, y los que están en un estrato social más bajo también.

De acuerdo a las características de la población, los estudiantes que trabajan para sostener su familia y que deben pagar sus estudios, tienen un nivel académico más bajo; sobre todo porque el tiempo dedicado al trabajo disminuye notablemente su tiempo dedicado al estudio. Como lo confirma Delgado 22 "existen estudiantes universitarios que tienen hogares conformados, con hijos y que, aunque no son los encargados del ingreso económico, se encargan de todas las labores del hogar, y en ocasiones de la crianza de sus hermanos menores; este fenómeno es más común en las mujeres". En el documento de la CEPAL se incluyen algunos datos de interés retomado por Rolando et $\mathrm{al}^{21}$ que menciona que "Los grupos más jóvenes alcanzan mejores grados de movilidad social que los de más edad".

En la teoría de movilidad social se plantean diversos factores que juegan un papel fundamental como lo es la educación y el deseo de mejorar el estatus económico en el que se encuentra, hecho mencionado por Rolando ${ }^{21}$ "paradigma también implicó el análisis del logro de estatus (status attainment).

Se indica que los factores referidos a la educación e ingresos/y mejor condicion económica; se rige bajo el supuesto de la existencia de una relación proporcional entre el logro educativo y el acceso a mejores posiciones en la estructura ocupacional, que determinan los niveles de ingreso, por tal razón los estudiantes de bajos recursos superan sus obstáculos y persisten con la finalidad de lograr una mejor calidad de vida y mejor posición social ${ }^{21}$.

Parsons ${ }^{23}$ presenta la característica grupal de la estratificación social, al sostener que la ocupación y el parentesco; representan un factor que estratifica a los individuos, generando una base de fundamental en la clasificación de los estratos sociales.

Los estudiantes universitarios de manera individual tienen "sus ideales, logros y metas educativas, se encuentran relacionado con abandonar la comunidad en donde se encuentran ubicados y de esta manera entrelazar las redes sociales y económicas que les permita adquirir un mejor estatus y comúnmente salarios más altos que los estándares locales" ${ }^{24}$. Además, es importante resaltar que la distribución de clases en los centros de estudios sobre movilidad social, es concebida como la segregación de los individuos entres las jerarquías sociales ${ }^{25,26}$.

Apoyando esta primicia, el pensamiento de los estudiantes universitarios y sus deseos de superación; se tiene que la medida de solución a este fenómeno se obtiene emigrando hacia el espacio en donde la educación y la obtención de logros se convierte en un trabajo remunerado y reconocido $0^{27,28}$. Apoyando esta primicia se resalta que algunos autores apuntan que en las zonas rurales y en los países desarrollados de América Latina, la experiencia de la educación media se convierte en opciones futuras de emigración por parte de los estudiantes, para un mejor futuro ${ }^{29}$.

\section{DECLARACIÓN SOBRE CONFLICTOS DE INTERÉS}

Los autores declaran no tener ningún conflicto de interés. No existe ningún interés financiero que constituya un conflicto de intereses, ya sea real, potencial y no se ha recibido beneficios como dinero o bienes de fuentes que tengan algún interés en los resultados de esta investigación.

\section{REFERENCIAS BIBLIOGRÁFICAS}

1. Tejedor F, García A. Causas del bajo rendimiento del estudiante universitario (en opinión de los profesores y alumnos). Propuestas de mejora en el marco del EEES Universidad de Salamanca. Revista de Educación. 2007; (342): 443-73.

2. Jörngården A, Weergen L, Von-Essen L. Measuring health-related quality of life in adolescents and young adults: Swedish normative data for the SF-36 and the HADS, and the influence of age, gender, and method of administration. Health and Quality of Life Outcomes. $2006 ; 4: 91: 1-10$

3. Zullig K, Valois R, Wanzer J. Adolescent distinction between quality of life and selfrated health in quality of life research. Healthand Quality of Life Outcomes. 2005; 3:64: 1-9

4. Ferrer R, Palmer R, Burge $\mathrm{S}$. The family contribution to health status: a population-level estimate. Ann Fam Med 2005; 3 (2):102-8.

5. Tseng HM, Rachel JF, Gandek B. Cultural issues in using the SF-36 health survey in Asia: results from Taiwan. Health and Quality of Life Outcomes .2003; 1:72-81.

6. Garbanzo G. Factores asociados al rendimiento académico en estudiantes universitarios, una reflexión desde la calidad de la educación superior pública. Universidad de Costa Rica San José, Costa Rica, Revista Educación. 2007; 31(1):43-63. 
7. Spínola H. Rendimiento académico y factores psicosociales en los ingresantes a la carrera de medicina - UNNE. Revista Paraguaya de Sociología. 1990; 78:143167.

8. Fergusson E, James D, Madeley L. Factors associated with success in medical school: systematic review of the literature. BMJ. 2002; 324:952-7.

9. Vélez-Van A, Roa NC. Factors associated with academic perfomance in medical students. En: PSIC. Educación Médica. 2005; 2(8):1-10.

10. Pérez A, Ramón J, Sánchez J. Análisis exploratorio de las variables que condicionan el rendimiento académico. Sevilla, España: Universidad Pablo de Olavide. 2000; 34:15-20.

11. Araya J, Sierra D. Influencia de factores de riesgo social en el origen de conductas delincuenciales. Índice de vulnerabilidad social- delictual comunal. Santiago de chile: División Seguridad Ciudadana; 2002.

12. Latiesa B. La deserción universitaria, desarrollo de la escolaridad en la enseñanza superior. Éxitos y Fracasos. Madrid, España: siglo XXI de España. 1992.

13. Salonava M, Cifre E, Grau R, Martínez I. Antecedentes de la autoeficacia en profesores y estudiantes universitarios: un modelo causal. Revista de Psicología del Trabajo y de las Organizaciones. 2005; 1-2(21): 159-176.

14. BID (Banco Interamericano de Desarrollo). Competitividad: el motor del crecimiento. Progreso económico y social en América Latina: Departamento de investigación. Informe 2001.

15. Quintero C, Lugo L, García H, Sánchez A. Validación del cuestionario KIDSCREEN-27 de calidad de vida relacionada con la salud en niños y adolescentes de Medellín, Colombia. Revista colombiana de Psiquiatría. 2011; 40(3):470-487.

16. O`Boyle C. The Schedule for the evaluation of individual Quality of life (SEIQoL). Internacional Journal of Mental Health, 1994; 23: 3-23.

17. Hernández R, Fernández C, Baptista P. Metodología de la investigación. Mexico: Ed. Mc Graw Hill; 2003.

18. Artunduaga M. Variables que influyen en el rendimiento académico. (Tesis doctoral). España; Universidad Complutense de Madrid; 2008.
19. Katschnig H, Freeman H, Sartorious N. Calidad de vida en los trastornos mentales. Barcelona: Masson;2000.

20. Vilagut G, Ferrer M, Rajmil L, Rebollo, P, Permanyer G, Quintana, JM, et al. El cuestionario de salud SF36 español: una década de experiencia y nuevos desarrollos. Gaceta sanitaria. 2005; 19(2):135-150.

21. Rolando F, León A, Atria R, Filgueira C, Méndez M, Gayo $\mathrm{M}$, et al. Estratificación y movilidad social en américa latina transformaciones estructurales de un cuarto de siglo. Santiago: LOM; 2007.

22. Delgado G. Condiciones escolares asociadas a la deserción en educación secundaria. Análisis a partir de dos casos en México. $2^{\circ}$ ed. México: Universitat de Girona; 2000.

23. Parsons T. Un enfoque analítico de la teoría de la estratificación social, en Ensayos de teoría sociológica. Buenos Aires: Ed. Paidós; 1967.

24. Corbett M. "'It was fine if you want to leave': Educational Ambivalence in a Nova Scotia Coastal Community, 1963-1998", Anthropology and Education Quarterly. 2004; 35(4): 451-471.

25. Goldthorpe JH. "Class and p o litics in ad vanced industrial societies", The Breakdown of Class Politics, T.N. Clark y S.M. Lipset (comps.). Washington, D.C: Woodrow Wilson C enter Press/The Johns Hopkins University Pres; 2001.

26. Ferrel F, Ortiz A, Forero L, Herrera M, Peña Y. Estilos de vida y factores sociodemográficos asociados en adolescentes escolarizados del Distrito de Santa Marta, Colombia. Duazary. 2014 Dic; 11 (2): 105 - 114

27. Pérez L. ¿Estudiar para emigrar o estudiar para transformar? Un acercamiento etnográfico a la erosión del significado de los estudios superiores como mecanismo meritocrático de movilidad social. 2010; 23(62): $1-26$.

28. Monterrosa A, Ulloque L, Carriazo S. Calidad del dormir, insomnio y rendimiento académico en Estudiantes de Medicina. Duazary. 2014 Dic; 11 (2): 85 - 97

29. Délano P, Larrañaga L. "El futuro de los jóvenes estudiantes rurales: iel campo o la ciudad?", Estudios pedagógicos. 1996; 22: 21-34.

Para citar este artículo: Brito-Jiménez IT, Palacio-Sañudo J. Calidad de vida, desempeño académico y variables sociodemográficas en Estudiantes universitarios de Santa Marta Colombia. Duazary. 2016 julio; 13 (2): 133 - 141 